\title{
Simulation in healthcare education: is it only about money?
}

\author{
T. Wegscheider ${ }^{1,2}$
}

\section{Keywords: Patient safety, Simulation and training}

\section{Editorial}

No musician would give a concert without having trained to perfection. No actor or actress would ever go on stage without knowing his or her role. Indeed, artist's training is really tough! Every movement during and even every bow at the end of their performance are trained to perfection. They invest plenty of time to achieve their one and only goal: to thrill their audience.

Imagine a theatre employing untrained musicians and actors! How long would this theatre survive? As in the world of art economic survival has always been also a matter of staff training, many theatres all over the world run well-equipped training facilities. So-called "rehearsal stages" provide the safe environment needed to try out new things, to gain self-confidence and to bring any performance to perfection. Training in the world of art is more than a successful concept - it is a successful culture.

What about this culture in healthcare? Do healthcare systems or hospital operators provide comparable safe environments? Are today's healthcare professionals fully enabled to train their skills before treating real patients? Are there enough facilities and programmes available to meet their needs for simulation-based skills training? And if not: Why is that? From the perspective of critically ill or perhaps even incurable persons all these questions reach a new dimension.

Nowadays more and more patients rightly refuse being used as a "learning object" [1]. Using simulation as an educational tool reduces risks for patients as well as for learners, allows repeated skills training and facilitates the

Correspondence: thomas.wegscheider@medunigraz.at

'Department of Anesthesiology and Intensive Care Medicine, Division of Special Anesthesiology, Pain-and Intensive Care Medicine, Medical University of Graz, Auenbruggerplatz 29, 8036 Graz, Austria

${ }^{2}$ Clinical Skills Center, Medical University of Graz, Graz, Austria transfer of classroom-knowledge to real situations [2]. But simulation is not only about technical skills performance. Simulation-based training paves the way for medical crews working together as medical teams even in critical situations. Simulation has the power to sensitize all people involved in patient care to the impact of human factors on care quality. Structured approaches and tools e.g., the life-saving ' 10 -seconds-for-10 minutes" principle [3] might be given a better chance to be put into daily practice by using simulation training and structured debriefing rather than using so-called "traditional" teaching methods.

Although significance and benefits of simulation-based training in the wide field of healthcare education have been undisputable for decades, in some respects even for centuries $[2,4]$, there are still blank spots in the core curricula of medical and paramedical educational institutions.

Simulation-based programmes in undergraduate education are often electives, reaching not all students. Moreover compulsory simulation-based training programmes are still very rare in postgraduate education.

Nevertheless strong efforts are made all over the world to promote simulation-based training as a vital part of patient-safety-training programmes. In 2009 the World Health Organization (WHO) published a Patient Safety Curriculum Guide for Medical Schools [5] followed by a Multi-professional Edition in 2011 [6]. Both documents emphasize the relevance of multiprofessional training using simulation to enhance patient safety $[5,6]$.

Although those responsible are mostly aware of the wide range of learning and teaching opportunities of simulation and its positive effects on patient safety, many educational institutions as well as hospital operators tend to show more hidden restraint than support. 
Two major reasons seem to be responsible for that unsatisfactory situation: First, costs for running simulation centres are often too high, so some institutions cannot provide an additional facility only dedicated to simulation purposes. Second, legal regulations lower the time that staff can use for skills training. Working and training times become more and more limited [1]. Of course, these points have to be taken into account by all of those responsible. But is there really no escape out of this dilemma?

A well-known saying goes "Where there's a will, there's a way".

If an institution cannot afford a simulation facility for lack of financial or infrastructural resources it's time to be creative and look for alternatives. Maybe the acquisition of a high-fidelity patient simulator exceeds the budget, so low-fidelity part task trainers should be considered a lessexpensive alternative [2]. If a hospital cannot provide highly equipped infrastructure for simulation purposes for lack of space, "in situ" simulation might be the solution to the problem. Using the "in situ" approach, trainees are trained in their real working environment, so no additional infrastructure is needed [1]. Keeping trainings focused and short might help dealing with the decreasing availability of personnel due to legal regulations.

As you can see, the saying mentioned above seems to prove itself to be true. But: What again about the blank spots in the core curricula of medical and paramedical schools?

What about health care professionals being not given the chance to train their skills to perfection before treating real patients? Are lacks of room and money really an excuse for patient-safety threatening passivity by those responsible? As shown above, there are actually many alternatives and with a little creativity there will be even more. Fortunately there seems to be a way! But is there a will? Do healthcare systems and hospital operators really know about the power of new teaching and training modalities like simulation-based training? Do they belief in the positive effects on employee satisfaction and are they aware of savings potentials of innovative educational interventions? Maybe some are not! Maybe some are still stuck in the traditional "see one, do one, teach one" concept.

Safety in Health as a new growing up journal aims to inform its readers about modern ways of education and training in healthcare. We intend to present a wide range of research in the field of healthcare education and will be happy about scientific contributions from all over the world. Together we are looking forward to the day when training in healthcare has become the same successful culture as it already is in other high-risk industries. Sometimes it only needs money but even more often it needs a cultural change. As the saying goes "Where there's a will, there's a way"!

\section{Competing interests}

The author declares that he has no competing interests.

Received: 10 September 2015 Accepted: 1 December 2015 Published online: 15 January 2016

\section{References}

1. Lois FJ, Pospiech AL, Van Dyck MJ, Kahn DA, De Kock MF. In the "in situ" simulation for teaching anesthesia residents a lower cost, feasible and satisfying alternative to simulation center? A 24 months prospective observationale study in a university hospital. Acta Anaesthesiol Belg. 2014; 65:61-71.

2. Maran NJ, Glavin RJ. Low- to high-fidelity simulation - a continuum of medical education? Med Educ. 2003;37:22-8.

3. Rall M, Glavin RJ, Flin R. The '10-seconds-for-10-minutes principle'. Why things go wrong and stopping them getting worse. Bull Royal Coll Anaes. 2008;51:2614-6.

4. Rosen KR. The history of medical simulation. J Crit Care. 2008;23:157-66.

5. World Health Organization. Patient safety curriculum guide for medical schools. Geneva: World Health Organization; 2009.

6. World Health Organization. Patient safety curriculum guide: multiprofessional edition. Geneva: World Health Organization; 2011.

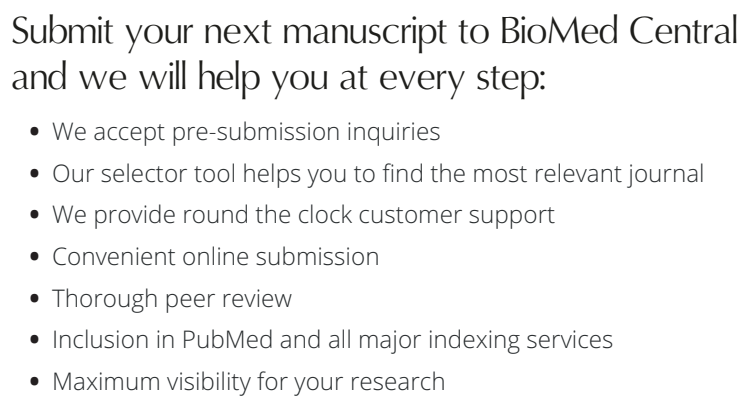

C Biomed Central 